\title{
Évaluation de la gestion des anticoagulants oraux en chirurgie de prothèse de hanche ou de genou
}

\author{
Baptiste Compagnon, MBBS $\cdot$ Remi Bouquerel, MD $\cdot$ Benjamin Le \\ Gaillard, MD · Antoine Strzelecki, MD - Christophe Pelletier, MD • \\ Mireille Houadec, MD • Gorka Usandizaga, MD • Edmundo Pereira \\ de Souza Neto, MD, PhD
}

Received: 13 March 2020/Revised: 19 August 2020/Accepted: 19 August 2020/Published online: 2 September 2020

(C) Canadian Anesthesiologists' Society 2020

Au rédacteur en chef,

Les anticoagulants oraux d'action directe (AOD) sont une classe médicamenteuse hétérogène en termes de recommandations d'utilisation et de profils pharmacologiques. ${ }^{1}$ Leur dosage n'est pas nécessaire mais peut s'avérer utile dans certaines situations. ${ }^{2}$

Entre le $1^{\text {er }}$ janvier 2017 et le 15 juillet 2019, nous avons colligé, rétrospectivement, chez les patients qui ont bénéficié d'une arthroplastie totale unilatérale primaire de la hanche (PTH) ou du genou (PTG), les données concernant les délais d'arrêts des AOD et leurs dosages plasmatiques. Nous avons répertorié les complications hémorragiques chirurgicales jusqu'à deux mois postopératoires, comprenant l'hématome du site opératoire ou la transfusion de concentré de globules rouges.

Conformément à la réglementation française, les études rétrospectives basées sur l'exploitation des données courantes de soins ne doivent pas être soumises à un comité éthique.

Les données des patients mineurs, opérés en urgence, subissant une arthroplastie secondaire ou sous antivitamine $\mathrm{K}$, n'ont pas été recueillies. Tous les patients ont été opérés par le même chirurgien.

B. Compagnon, MBBS $(\varangle) \cdot$ R. Bouquerel, MD .

B. Le Gaillard, MD - A. Strzelecki, MD - C. Pelletier, MD

M. Houadec, MD - E. Pereira de Souza Neto, MD, PhD

Département d'anesthésie, Centre Hospitalier de Montauban,

Montauban, France

e-mail: baptiste.compagnon@hotmail.fr

G. Usandizaga, MD

Département de chirurgie orthopédique et traumatologie, Centre

Hospitalier de Montauban, Montauban, France
Les délais d'arrêt des AOD variaient de trois à cinq jours, comme le suggère le Groupe d'Intérêt en Hémostase Périopératoire. ${ }^{3}$ Le dosage plasmatique spécifique de l'AOD a été fait selon la méthode chromogénique.

Sept cent soixante dossiers ont été analysés, dont 307 PTH et 453 PTG. Quarante-cinq patients prenaient un AOD (23 rivaroxaban, 19 apixaban et 3 dabigatran). Trente-six pourcent des sujets sous AOD ont bénéficié d'un dosage de leur AOD avant la chirurgie, à la demande de l'anesthésiste lors de la consultation. Tous les dosages réalisés étaient inférieurs à $30 \mathrm{ng} \cdot \mathrm{mL}^{-1}$. Nous avons recueilli les données de 122 patients avec complications hémorragiques, soit un taux global de $16 \%$, dans les groupes PTH et PTG, dont 111 sans AOD et 11 avec AOD. Il n'y a pas de différence entre les groupes PTH et PTG, avec et sans AOD, pour les taux de complications hémorragiques (Tableau 1).

Dans le groupe PTH, il n'y a pas de différence entre les deux sous-groupes, avec et sans AOD, concernant l'âge, le débit de filtration glomérulaire (DFG) et le type d'anesthésie reçu. L'indice de masse corporelle (IMC) est plus bas dans le groupe avec AOD $(P=0,047)$.

Dans le groupe PTG, il n'y a pas de différence entre les deux sous-groupes concernant l'âge, l'IMC et le DFG. Cependant, les patients sous AOD ont bénéficié de davantage d'anesthésie générale $(5 / 7$, soit $71 \%)$ par rapport au groupe sans AOD $(17 / 71$, soit $24 \%)(P=$ 0,017 ). Les patients de statut III selon l'ASA (American Society of Anesthesiologists) prédominaient dans le groupe avec AOD $(P<0,001)$.

Nous n'avons retrouvé aucune complication hémorragique liée à l'anesthésie péri- médullaire dans les conditions d'arrêts des AOD variant de trois à cinq jours.

Aucune corrélation n'a été retrouvée entre le dosage plasmatique de l'AOD et son délai d'arrêt. 
Tableau 1 Comparaison des taux de complications hémorragiques des patients des groupes PTH et PTG sans et avec AOD

\begin{tabular}{llc}
\hline & $\begin{array}{l}\text { Patients avec complications hémorragiques } \\
n=\mathbf{1 2 2}\end{array}$ & Avec AOD \\
\cline { 2 - 3 } & Sans AOD & $30,7 \%(4)$ \\
\hline PTH \% $(n):$ & $13,6 \%(40)$ & $21,8 \%(7)$ \\
- AOD + $(n=13)$ & & \\
- AOD - $(n=294)$ & $16,9 \%(71)$ & 0,099 \\
PTG \% $(n):$ & \\
- AOD $+(n=32)$ \\
- AOD - $(n=421)$ \\
\hline$+=$ avec; $-=$ sans; AOD = anticoagulants oraux d'action directe; PTG = prothèse totale de genou; PTH = prothèse totale de hanche. Les données
\end{tabular}
ont été analysées selon le test de Fisher

Notre étude présente des limites notamment liées au faible nombre de dosages réalisés et au caractère rétrospectif avec des possibles biais de sélection. Les patients opérés d'une PTG sous AOD ont bénéficié de davantage d'anesthésie générale par rapport au groupe sans AOD. Cela peut s'expliquer par une potentielle crainte par l'anesthésiste de l'hématome péri-médullaire. En effet, les sociétés savantes françaises n'apportent que des suggestions, et non des recommandations, sur la gestion de ces médicaments avant une rachianesthésie. ${ }^{3}$

Nos patients sous AOD semblent avoir le même taux de complications hémorragiques chirurgicales que ceux sans AOD. D'autres études seront nécessaires afin d'explorer certaines zones d'ombres des AOD : la variabilité interindividuelle d'élimination et l'incidence des hématomes péri-médullaires post-anesthésie neuraxiale.

\section{Déclaration Aucune.}

Déclaration de financement Aucune.

Editorial responsibility Cet article a été traité par Dr Étienne de Médicis, rédacteur de la langue française, Journal canadien d'anesthésie.

\section{Références}

1. Agence nationale de sécurité du médicament (ANSM). Les anticoagulants en France en 2014 : état des lieux, synthèse et surveillance - Avril 2014. Disponible à l'adresse URL: https:// ansm.sante.fr/content/download/61981/795269/version/2/file/ANS M-rapport_NACOs-avril+2014.pdf (consulté août 2020).

2. Konkle BA. Direct oral anticoagulants: monitoring anticoagulant effect. Hematol Oncol Clin North Am 2016; 30: 995-1006.

3. Groupe d'Intérêt en Hémostase Périopératoire (GIHP). Gestion des Anticoagulants Oraux Directs pour la chirurgie et les actes invasifs programmés : propositions réactualisées du Groupe d'Intérêt en Hémostase Périopératoire (GIHP). Septembre 2015. Disponible à l'adresse URL: https://sfar.org/espace-professionelanesthesiste-reanimateur/outils-professionnels/boite-a-outils/reactualisation-des-propositions-du-gihp-pour-la-gestion-perioperatoire des-patients-sous-aod-pour-un-acte-programme/ (consulté août 2020).

Publisher's Note Springer Nature remains neutral with regard to jurisdictional claims in published maps and institutional affiliations. 